\title{
EXTRAÇÃO ASSISTIDA POR ULTRASSOM PARA DETERMINAÇÃO DE LIPÍDEOS EM ALIMENTOS: UM EXPERIMENTO DE LABORATÓRIO
}

\author{
Bruno L. Ferreira ${ }^{a}$, Junior V. Beik ${ }^{\mathrm{b}}$, Salvino J. Z. Alves ${ }^{\mathrm{c}}$, Flavia A. Henrique ${ }^{\mathrm{b}}$, Elenise Sauer ${ }^{\mathrm{d}}$, Cesar A. Chornobai ${ }^{\mathrm{d}}$, Simone \\ Bowles $^{\mathrm{b}}$ e Eduardo S. Chaves ${ }^{\mathrm{c}, *,(1)}$ \\ aDepartamento de Ciência e Tecnologia de Alimentos, Universidade Federal de Santa Catarina, 88034-000 Florianópolis - SC, Brasil

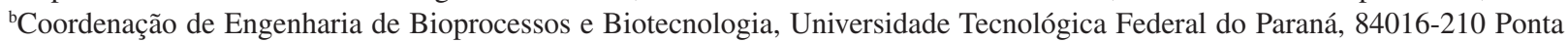 \\ Grossa - PR, Brasil \\ 'Departamento de Química, Universidade Federal de Santa Catarina, 88040-970 Florianópolis - SC, Brasil \\ ${ }^{\mathrm{d}}$ Departamento de Engenharia Química, Universidade Tecnológica Federal do Paraná, 84016-210 Ponta Grossa - PR, Brasil
}

Recebido em 26/03/2020; aceito em 01/06/2020; publicado na web em 15/07/2020

\begin{abstract}
ULTRASOUND ASSISTED EXTRACTION FOR LIPIDS DETERMINATION IN FOOD: A LABORATORY EXPERIMENT. The ultrasound-assisted extraction (UAE) is an alternative approach for the total lipid content determination in food samples. The high extraction efficiency, being less time-consuming and with the possibility of using less toxic solvents are the main advantages of UAE. It is, thus, an excellent alternative to be applied as a laboratory experiment for undergraduate students. The UAE procedure was optimized and the extraction parameters, such as extractor solvent, time and frequency of the ultrasound, were evaluated. The accuracy of the UAE procedure was evaluated by comparing the obtained results with those found by the traditional lipids extraction approach. The results showed that the lipids content found in the analyzed samples by using UAE were in agreement with the results obtained by the traditional lipids extraction. Therefore, the optimized UAE procedure was proposed as a laboratory experiment to 15 groups of undergraduate students to introduce the subjects of an alternative approach for total lipids determination in food samples. The results obtained showed that $80 \%$ of the student groups found results for total lipid contents in agreement with those found by traditional lipids extraction method. The proposed UAE procedure as a laboratory experiment proved to be simple and easy to be applied to undergraduate students.
\end{abstract}

Keywords: ultrasound-assisted extraction; lipids extraction; laboratory experiment

\section{INTRODUÇÃO}

O ensino de química desenvolve e articula uma rede complexa de conceitos científicos, sendo a experimentação uma das suas bases de sustentação. ${ }^{1}$ Para o ensino de ciência, Marcelo Giordan (1999) ${ }^{2}$ destaca a importância da abordagem experimental no desenvolvimento do conhecimento sistemático, pois o conhecimento se desenvolve, preferencialmente, nos entremeios da investigação. ${ }^{2}$ Neste sentido, é preciso conduzir práticas experimentais no ensino superior que vislumbrem a atenção e a percepção dos estudantes aliadas ao uso de técnicas emergentes. Assim, a Química Verde se preocupa em conduzir ações científicas e/ou processos industriais ambientalmente amigáveis, abarcando os anseios de um mundo em constante transformação. ${ }^{3}$

Nas práticas de laboratório é essencial fomentar a capacidade crítica dos estudantes de graduação, propondo metodologias alternativas às tradicionais, utilizando solventes mais seguros, buscando eficiência energética, elevadas taxas de extração de espécies químicas de interesse, baixo consumo de reagente e o desenvolvimento de uma química segura visando a prevenção de acidentes. ${ }^{3}$

A extração de lipídeos em diferentes matrizes teve seu avanço com o desenvolvimento de um aparelho pensado por Franz Von Soxhlet, por este motivo é conhecido como extração Soxhlet. A extração de lipídeos totais é baseada na transferência física destes para o(s) solvente(s) extrator(es), o qual está em contato com a amostra. ${ }^{4,5}$ Para a extração dos lipídeos, a amostra fica em contato com o solvente, o qual é adicionado constantemente no sistema, e dependendo do solvente extrator, a temperatura de extração é relativamente alta. Este procedimento de extração foi desenvolvido em 1879, sendo este

\footnotetext{
*e-mail: eduardo.chaves@ufsc.br
}

amplamente utilizado até os dias atuais. ${ }^{6}$ Entretanto, as limitações deste método de extração estão relacionadas ao volume excessivo de solvente, ao longo tempo e a necessidade de temperaturas elevadas para extração, as quais fazem deste um experimento laborioso e perigoso para ser aplicado em aulas práticas de laboratório. Nesse sentido, procedimentos alternativos de extração, tal como a extração assistida por ultrassom (UAE), que permitam uma extração rápida, eficiente e ambientalmente amigável, têm sido utilizados. ${ }^{7-10}$

A UAE é considerada uma técnica ambientalmente amigável para indústria de alimentos, principalmente, devido às características atribuídas ao ultrassom. Nos processos de extração envolvendo ultrassom, ondas mecânicas que se propagam através do meio em ciclos de compressão e rarefação em frequências entre $20 \mathrm{kHz}$ e $100 \mathrm{kHz}$, promovem o fenômeno chamado de cavitação. Esse fenômeno envolve a nucleação, o crescimento e o colapso de microbolhas nos líquidos, acarretando em um aumento pontual da temperatura e da pressão no meio por onde as ondas se propagam, bem como a formação de microjatos. Os microjatos são responsáveis pela erosão e/ou ruptura das partículas sólidas presentes no meio ${ }^{7,9,10}$, proporcionando uma boa eficiência de extração. Otimizando parâmetros como a frequência, potência e o tempo de ultrassom, é possível obter extrações de espécies químicas de interesse com recuperações semelhantes às dos métodos clássicos.

A UAE tem sido utilizada para extração de lipídeos de Chlorella vulgaris com líquidos iônicos, ${ }^{11}$ de óleo de soja com uso de hexano e isopropanol ${ }^{12}$ e extração em meio aquoso de óleo de arroz. ${ }^{13}$ Além disso, a UAE, associada com a extração por Soxhlet, tem sido utilizada para aumentar a eficiência da extração de óleos em sementes oleaginosas. ${ }^{14}$ Entretanto, apesar das vantagens associadas à utilização da UAE, este processo ainda é pouco aplicado para extração de lipídeos 
totais em amostras industrializadas, e tampouco existem métodos oficiais utilizando essa tecnologia para a extração dos mesmos.

A utilização de procedimentos alternativos para extração de lipídeos usando UAE em experimentos de laboratório é limitada. Assim, experimentos de laboratório que proporcionem a extração eficiente dos lipídeos em amostras de alimentos e que sejam ambientalmente amigáveis são fundamentais para ampliar o conhecimento dos estudantes. Os conceitos e a teoria envolvida no presente trabalho podem ser considerados simplificados e acessíveis para estudantes de graduação em início de curso, servindo para demonstrar os princípios básicos de análise química, utilizando UAE para o preparo de amostra, processos de extração e comparações dos resultados com os obtidos por métodos oficiais para determinação de lipídeos totais em amostras alimentares. Assim, um procedimento para determinação do teor de lipídeos totais em amostras de alimentos usando UAE foi proposto para posterior aplicação como experimento de laboratório para alunos em nível de graduação de cursos como Engenharia Química e Tecnologia de Alimentos. Este experimento foi proposto com o objetivo de demonstrar/investigar o processo de extração dos lipídeos usando UAE visando determinar o teor total de lipídeos em amostras de bolachas do tipo água e sal. Os resultados obtidos pelos estudantes foram posteriormente comparados com os obtidos pelos métodos oficiais de extração de lipídeos.

\section{PARTE EXPERIMENTAL}

\section{Reagentes e amostras}

O hexano (Cinética, Brasil), 1-propanol (Synth, Brasil), isopropanol (Synth) e éter etílico (Synth) foram avaliados para a extração de lipídeos por UAE. Foram analisadas duas amostras de bolacha, do tipo água e sal, adquiridas no comércio local do município de Ponta Grossa - Paraná.

\section{Instrumentação}

As amostras de bolacha foram devidamente homogeneizadas em processador tipo mixer (Britânia) e peneiradas, garantindo um tamanho de partícula $<1,0 \mathrm{~mm}$. Uma balança analítica (MARK 205A, BEL Engineering) foi utilizada para pesagens das amostras e das frações lipídicas após a extração. Uma estufa com circulação e renovação de ar (SL 102, SOLAB) foi utilizada para a secagem das vidrarias e dos extratos orgânicos contendo os lipídeos, e também para determinação da umidade das amostras. Para extração foram utilizados agitador do tipo vórtex (K45-2820, KASVI) e banhos ultrassônicos (USC-1400 40 kHz, 135 W e USC1650-A 25 kHz 120 W, UNIQUE). A centrifugação foi realizada em centrífuga (Excelsa Baby II 206-R, FANEM). Para verificar a exatidão do procedimento proposto, a extração foi realizada por extrator de lipídeos, método Goldfish (TECNAL).

\section{Procedimento}

\section{Determinação da umidade e verificação da exatidão}

A umidade das amostras de bolacha foi determinada conforme o método 012/IV do Instituto Adolfo Lutz utilizando secagem direta até peso constante em estufa a temperatura de $105^{\circ} \mathrm{C}$. ${ }^{4}$ Para verificação da exatidão, o teor de lipídeos totais nas amostras de bolacha foi determinado com base no método 032/IV do Instituto Adolfo Lutz, utilizando hexano como solvente extrator. ${ }^{4}$ A avaliação estatística dos resultados foi realizada por meio da aplicação do teste de Tukey, utilizando o software SASM-AGRI, ${ }^{15}$ e do teste-t, ${ }^{16}$ ambos considerando um nível de confiança de $95 \%(\mathrm{p}<0,05)$.

\section{Extração assistida por ultrassom}

Uma alíquota de 1,0 g de amostra foi diretamente pesada em um tudo de ensaio e, posteriormente, adicionada de 5,0 $\mathrm{mL}$ de hexano. Os tubos contendo as amostras foram tampados, agitados em vórtex por 20 segundos e submetidos a ação do ultrassom por 20 minutos. As amostras foram então centrifugadas a uma rotação de $5000 \mathrm{rpm}$ por 3 minutos e o sobrenadante, contendo a fração lipídica, foi transferido cuidadosamente para um béquer previamente pesado. Após a extração, as amostras foram submetidas a duas etapas de lavagem, sendo que em cada uma destas etapas uma alíquota de 5,0 mL de hexano foi adicionada ao resíduo da extração, submetidas à ação do ultrassom por 5 minutos e, posteriormente, o solvente foi separado. O solvente das extrações foi evaporado e os béqueres, contendo a fração lipídica, foram mantidos em estufa a $100 \pm 5{ }^{\circ} \mathrm{C}$ por pelo menos 2 horas. Os béqueres foram retirados da estufa, mantidos em dessecador até atingirem a temperatura ambiente, e pesados. Este procedimento está esquematicamente ilustrado na Figura 1.

Todas as análises foram realizadas em triplicata, o teor total de lipídeos nas amostras foi calculado conforme a equação 1 e os resultados expressos como média \pm desvio padrão $(n=3)$.

$$
\% \text { lipídeos totais }=\left(\frac{M_{\text {béquer }+ \text { lipideos }}-M_{\text {béquer vazio }}}{M_{\text {amostra }}}\right) \times 100
$$

onde $M_{\text {béquer + lipideos }}$ é a massa em gramas do béquer contendo os lipídeos extraídos após a evaporação do solvente extrator, $M_{\text {béquer vazio }}$ é a massa em gramas do béquer previamente pesado e $M_{\text {amostra }}$ é a massa em gramas da amostra seca.

\section{Segurança e resíduos}

Os processos de extração dos lipídeos, separação do sobrenadante e evaporação do hexano devem ser realizados em capela e os alunos devem usar os equipamentos de proteção individual, tais como guarda-pó, óculos de segurança e luvas. O hexano é um solvente potencialmente tóxico, tem caráter carcinogênico, pode provocar irritação na pele e em vias respiratórias, devendo ser manuseado com cuidado. Além disso, o hexano apresenta toxicidade para o meio ambiente. Assim, após a utilização, este deve ser descartado corretamente em recipiente previamente identificado ou destinado para o seu reaproveitamento após purificação. Ao colocar ou retirar as amostras da estufa, os alunos devem estar atentos para evitar queimaduras, sendo as garras e pinças instrumentos essenciais para esta etapa do processo.

\section{RESULTADOS E DISCUSSÃO}

\section{Otimização da extração assistida por ultrassom}

A extração de lipídeos é geralmente realizada com hexano e/ ou éter etílico, que devido as suas características físico-químicas (ponto de ebulição, pressão de vapor e polaridade), permitem uma boa eficiência na extração. ${ }^{12,17}$ Entretanto, álcoois como o isopropanol e etanol também têm sido utilizados para extração de óleos vegetais. ${ }^{12}$ Estes, apesar de apresentarem uma menor afinidade pelas moléculas de lipídeos, são seguros, possuem baixo potencial tóxico ${ }^{12}$ e suas características físico-químicas podem possibilitar a extração dos lipídeos. Neste sentido, hexano, isopropanol, 1-propanol e éter etílico foram avaliados como potenciais solventes para extração dos lipídeos nas amostras de bolacha (Figura 2A).

Os resultados obtidos indicam que os solventes extratores éter etílico e hexano proporcionaram uma maior eficiência de extração, já que os maiores teores de lipídeos foram obtidos com o uso destes 

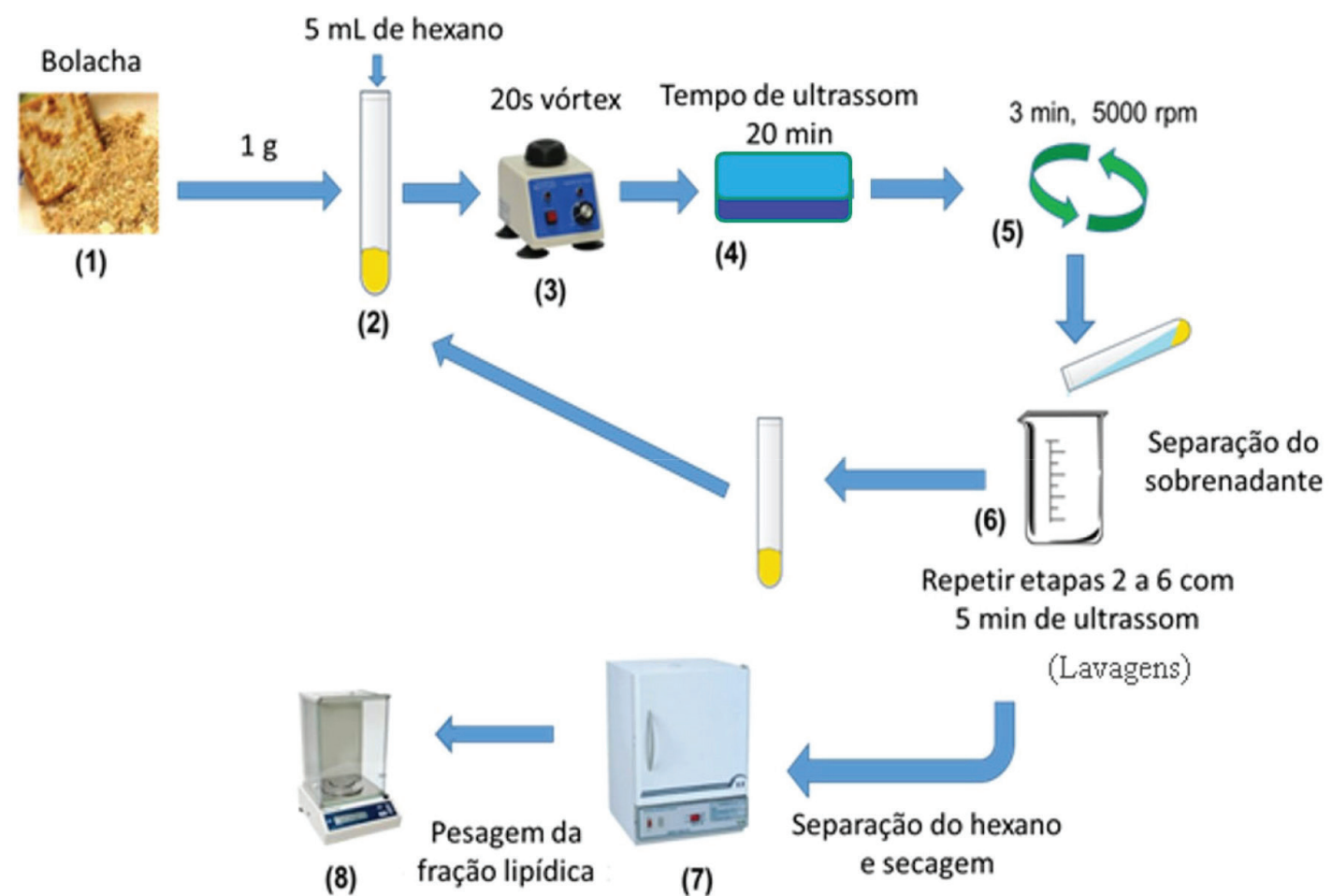

(Lavagens)

Figura 1. Representação esquemática do procedimento otimizado para extração assistida por ultrassom para determinação de lipídeos totais em bolachas

solventes. Estes solventes possuem baixa polaridade, consequentemente apresentam alta afinidade com as moléculas dos lipídeos presentes na amostra, facilitando o processo de extração. $\mathrm{O}$ isopropanol e o propanol também possibilitaram a extração dos lipídeos, porém com uma eficiência de extração relativamente menor quando comparada as extrações com éter e/ou hexano. A eficiência de extração está relacionada com a afinidade do solvente extrator com os lipídeos presentes na amostra. ${ }^{12}$ Neste caso, a afinidade dos álcoois pelas moléculas de lipídeos é relativamente menor que a afinidade do hexano e do éter por estas moléculas.

Considerando que a aplicação do método proposto será em experimentos de laboratório, a utilização do éter etílico como solvente extrator pode não ser adequada, devido sua alta volatilidade, inflamabilidade e baixo ponto de ebulição. A utilização do éter etílico, mesmo que realizada em capela de exaustão pode promover uma significativa liberação de vapores no laboratório, o que pode tornar o ambiente altamente insalubre para alunos e professores. Assim, optou-se por utilizar hexano como solvente extrator, pois além de proporcionar uma boa eficiência de extração dos lipídeos, possui maior ponto de ebulição e menor pressão de vapor quando comparado ao éter etílico.

A influência da frequência e potência do ultrassom foi também avaliada e os resultados estão apresentados na Figura 2B. Os resultados foram obtidos a partir da utilização de dois banhos ultrassônicos, um com frequência de $25 \mathrm{kHz}$ e potência de $120 \mathrm{~W}$ e outro com frequência de $40 \mathrm{kHz}$ e $135 \mathrm{~W}$ de potência. A potência do ultrassom pode influenciar no processo de extração, pois a utilização de potencias elevadas favorece o processo de cavitação, consequentemente, aumentam a difusão do analito e a turbulência interfacial, proporcionando um aumento na eficiência de extração. ${ }^{18,19}$ Adicionalmente, o tamanho das bolhas geradas pela cavitação e os processos de transferência de massa estão diretamente relacionados com a frequência do ultrassom. De modo geral, quanto menor a frequência maiores serão as microbolhas de cavitação e mais intensa é a implosão, o que pode aumentar a eficiência dos processos de
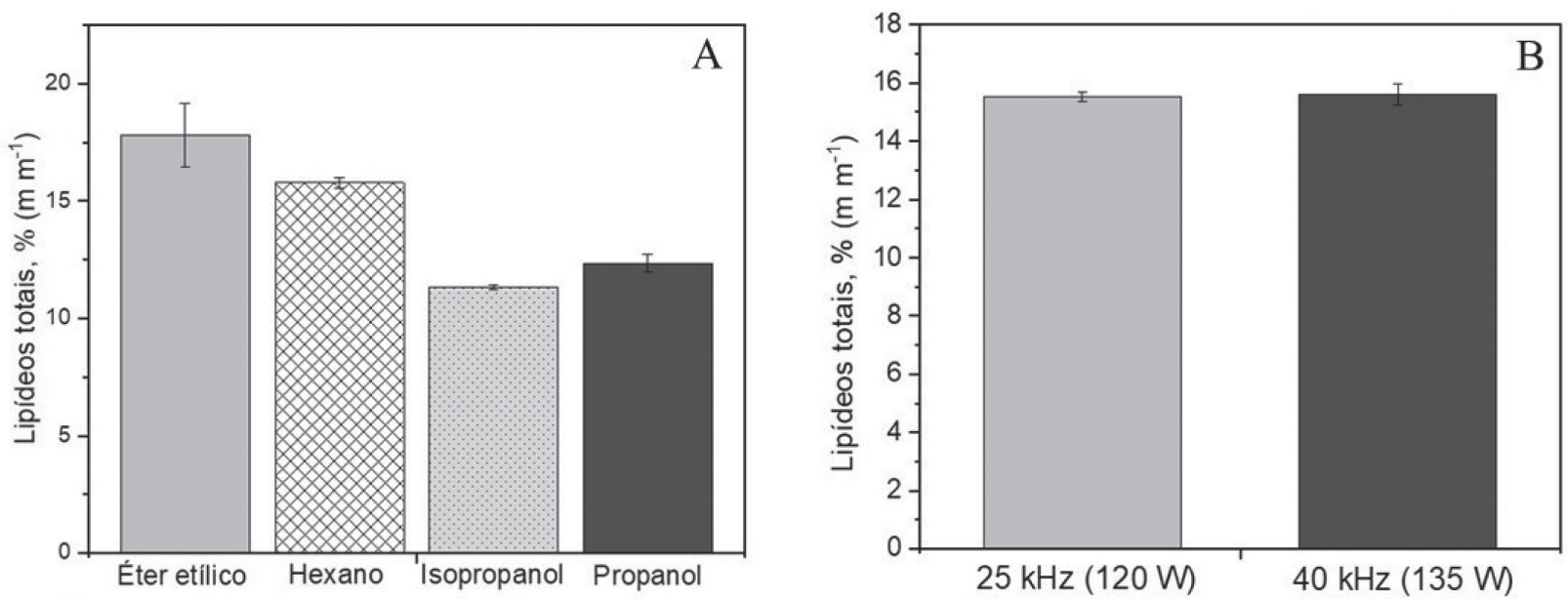

Figura 2. (A) Efeito do solvente extrator e (B) influência da frequência e potência do ultrassom na extração dos lipídeos em amostras de bolacha do tipo água $e$ sal. Tempo de extração em ultrassom de $10 \mathrm{~min}$, massa de amostra: 1,0 g e volume de solvente extrator: 5,0 mL. As barras representam o desvio padrão ( $\mathrm{n}=3$ ) 
extração. ${ }^{20}$ Entretanto, observou-se que para a amostra de bolacha utilizada para as otimizações, as concentrações de lipídeos totais obtidas com uso do banho ultrassônico de $25 \mathrm{kHz}(120 \mathrm{~W})$ e $40 \mathrm{kHz}$ $(135 \mathrm{~W})$ foram muito semelhantes, sendo estas de 15,53 $\pm 0,15$ e $15,61 \pm 0,38 \%\left(\mathrm{~m} \mathrm{~m}^{-1}\right)$, respectivamente. $\mathrm{O}$ teste $\mathrm{F}$ indicou que as variâncias dos resultados obtidos foram estatisticamente equivalentes $\left(\mathrm{F}_{\text {calculado }}=3,50<\mathrm{F}_{\text {crítico }}=19,0\right)$ para $\mathrm{p}<0,05$. Assim, aplicando o teste-t para duas amostras, presumindo variâncias equivalentes entre os resultados obtidos com banho de $25 \mathrm{kHz}$ e de $40 \mathrm{kHz}$, observou-se que os resultados dos lipídeos obtidos após extração não apresentaram diferença significativa $\left(\mathrm{t}_{\text {calculado }}=0,72<\mathrm{t}_{\text {crítico }}=2,78\right)$. Assim, os experimentos subsequentes foram realizados com o banho ultrassônico de $40 \mathrm{kHz}$.

O tempo de extração foi avaliado até 30 min de exposição ao ultrassom e os resultados estão apresentados na Figura 3. Observase que as extrações foram significativas após 5 min de exposição ao ultrassom. Entretanto, os resultados obtidos com 20 min de extração apresentaram um valor médio para o teor de lipídeos ligeiramente superior aos demais tempos avaliados, além de proporcionar um baixo desvio padrão. O teste de Tukey indicou que, para os tempos avaliados, a eficiência de extração não foi significativamente diferente. O tempo de $20 \mathrm{~min}$ foi selecionado para extração dos lipídeos nas amostras de bolacha, pois além de proporcionar teor de lipídeos ligeiramente maior, este é um tempo satisfatório para ser aplicado em aulas práticas de laboratório.

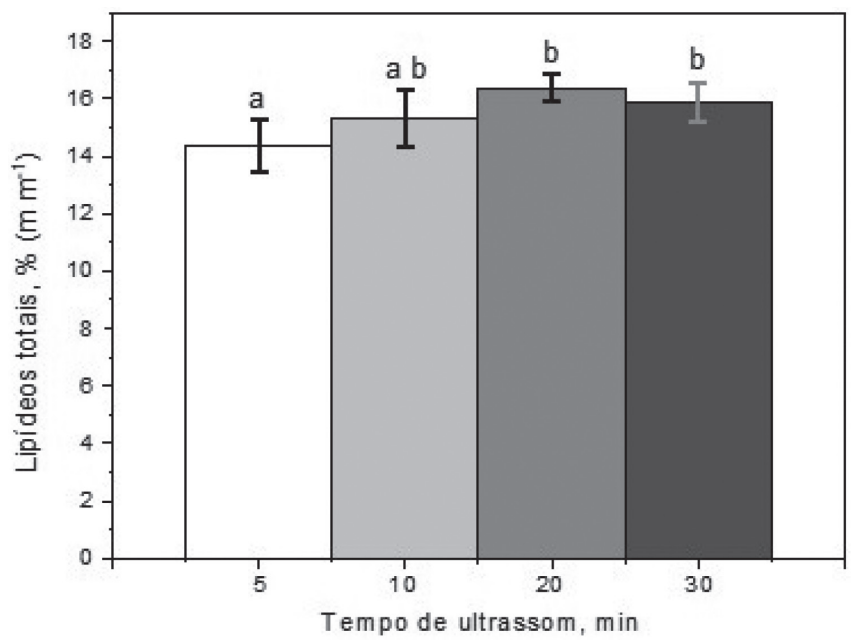

Figura 3. Efeito do tempo de ultrassom na extração de lipídeos em amostras de bolacha do tipo água e sal. Massa de amostra: 1,0 g e volume de solvente extrator: 5,0 mL. Barras de erro representam o desvio padrão $(n=3)$. Letras diferentes indicam uma diferença estatística com um nível de confiança de 95\% (Teste de Tukey)

A determinação de lipídeos totais em amostras de alimentos é tradicionalmente realizada seguindo o método oficial que usa extração utilizando os métodos de Soxhlet ou Goldfish. Assim, a exatidão do método proposto foi avaliada por meio da comparação dos resultados obtidos usando a UAE com os resultados obtidos utilizando o método 032/IV do Instituto Adolfo Lutz. ${ }^{4}$ Adicionalmente, foram avaliados os resultados obtidos após $20 \mathrm{~min}$ de ação do ultrassom combinados com as duas etapas de lavagens, com 5,0 mL de hexano e utilizando 5 min adicionais de exposição ao ultrassom em cada etapa (conforme descrito na parte experimental). Os resultados obtidos pelo método proposto para determinação de lipídeos totais em amostras de bolacha, com e sem as etapas adicionais de lavagens, assim como os resultados obtidos pelo método do IAL, estão apresentados na Figura 4.

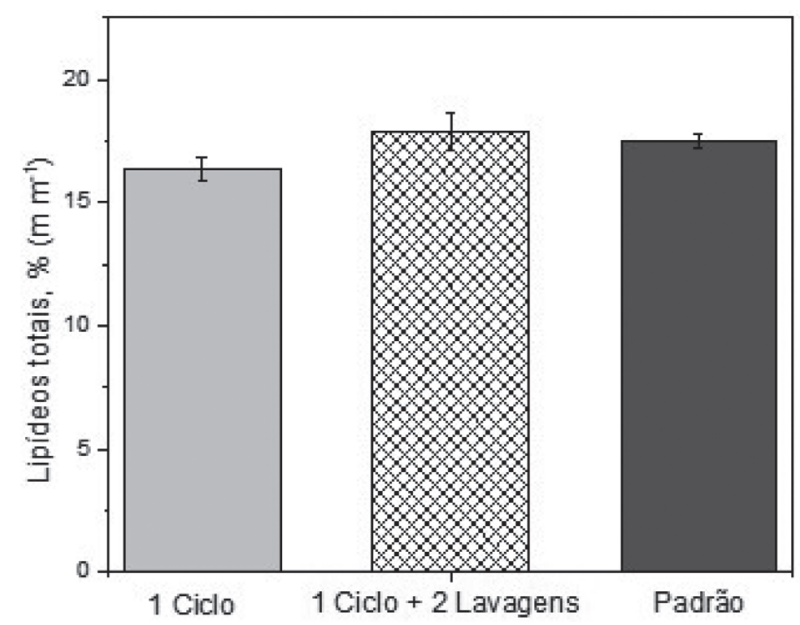

Figura 4. Verificação da exatidão do método proposto para determinação de lipídeos totais em amostras de bolacha usando extração assistida por ultrassom. Massa de amostra: 1,0 g, volume de solvente extrator: 5,0 mL, tempo de extração de 20 min. Lavagens com tempo ultrassom de 5 min e comparação com método padrão (032/IV do Instituto Adolfo Lutz). As barras representam o desvio padrão $(n=3)$

Considerando a concentração obtida pelo método padrão como valor de referência utilizou-se o teste t de Student para avaliação estatística dos resultados. Os valores de t calculados para a extração sem as etapas de lavagem ( 1 ciclo) e com as duas etapas adicionais de lavagem ( 1 ciclo + 2 lavagens) foram respectivamente 4,21 e 0,86. Estes valores são menores que o $t_{\text {crítico }}=4,30$ para dois graus de liberdade, o que indica que não há diferença significativa entre os resultados obtidos com a UAE e os obtidos pelo método do IAL. Entretanto, a extração com as lavagens adicionais proporcionou resultados mais próximos dos obtidos com o método do IAL. Além disso, devemos considerar que a separação do sobrenadante contendo a fração lipídica é realizada de modo simples e sem filtração, apenas vertendo o extrato do tubo para o béquer após uma etapa rápida de centrifugação. Neste sentido, as etapas adicionais de lavagem podem minimizar as potenciais interferências e resultados subestimados. Ainda, as etapas adicionais com lavagens usando $5 \mathrm{~min}$ de ultrassom podem ser fundamentais para reduzir os erros experimentais durante a realização de uma prática de laboratório. Nesse sentido, optou-se por realizar as extrações com 20 min de ultrassom seguidas das duas lavagens adicionais com o hexano.

\section{Aplicação do método como experimento de laboratório}

Após essas otimizações o procedimento foi proposto como experimento de laboratório com objetivo de introduzir aos estudantes de graduação de diferentes cursos, tais como Química, Engenharia Química, Tecnologia de Alimentos etc, os conceitos de análise química quantitativa, Química Verde e da UAE, usando procedimentos alternativos aos convencionais para extração de lipídeos totais em amostras de alimentos. Os métodos convencionais de extração dos lipídeos são laboriosos, demandam um tempo longo de análise e requerem o uso de grandes volumes de solventes extratores. Assim, durante a realização do experimento, os fundamentos da extração convencional de lipídeos conforme os métodos oficiais foram apresentados e as vantagens e limitações foram discutidas fomentando o debate para práticas alternativas eficientes, com menor custo energético. Posteriormente, procedimentos alternativos envolvendo UAE, visando a redução do tempo, o uso de reagente e geração de resíduos foram também abordadas. Além disso, usando o contexto do experimento proposto, os fundamentos UAE bem como as suas aplicações em diferentes áreas foram discutidos. 
O experimento foi aplicado para três turmas, com alunos do Curso de Engenharia Química e Tecnologia de Alimentos, totalizando 15 grupos. Os estudantes desenvolveram o experimento proposto em grupos de 4 a 5 alunos em aulas práticas de laboratório com tempo total variando entre 3 e 4 horas-aula. Cada grupo recebeu aleatoriamente uma amostra de bolacha triturada e seca, com os valores de umidade e de lipídeos totais obtidos previamente por meio do método oficial do IAL. As amostras utilizadas nos experimentos de laboratório foram de bolacha do tipo água e sal, codificadas como amostra $1 \mathrm{C}$ e $2 \mathrm{~A}$ contendo teores de lipídeos de $12,47 \pm 0,57 \%$ e $11,15 \pm 0,99 \% \mathrm{~m} \mathrm{~m}^{-1}$ (média \pm intervalo de confiança de $95 \%$, $\mathrm{n}=3$ ) respectivamente.

Os resultados obtidos pelos diferentes grupos de alunos estão apresentados na Figura 5. As barras representam o valor médio \pm desvio padrão do teor de lipídeos totais obtidos por cada grupo de estudantes e a linha pontilhada ilustra o valor de referência \pm intervalo de confiança com $95 \%$, obtido por extração convencional.
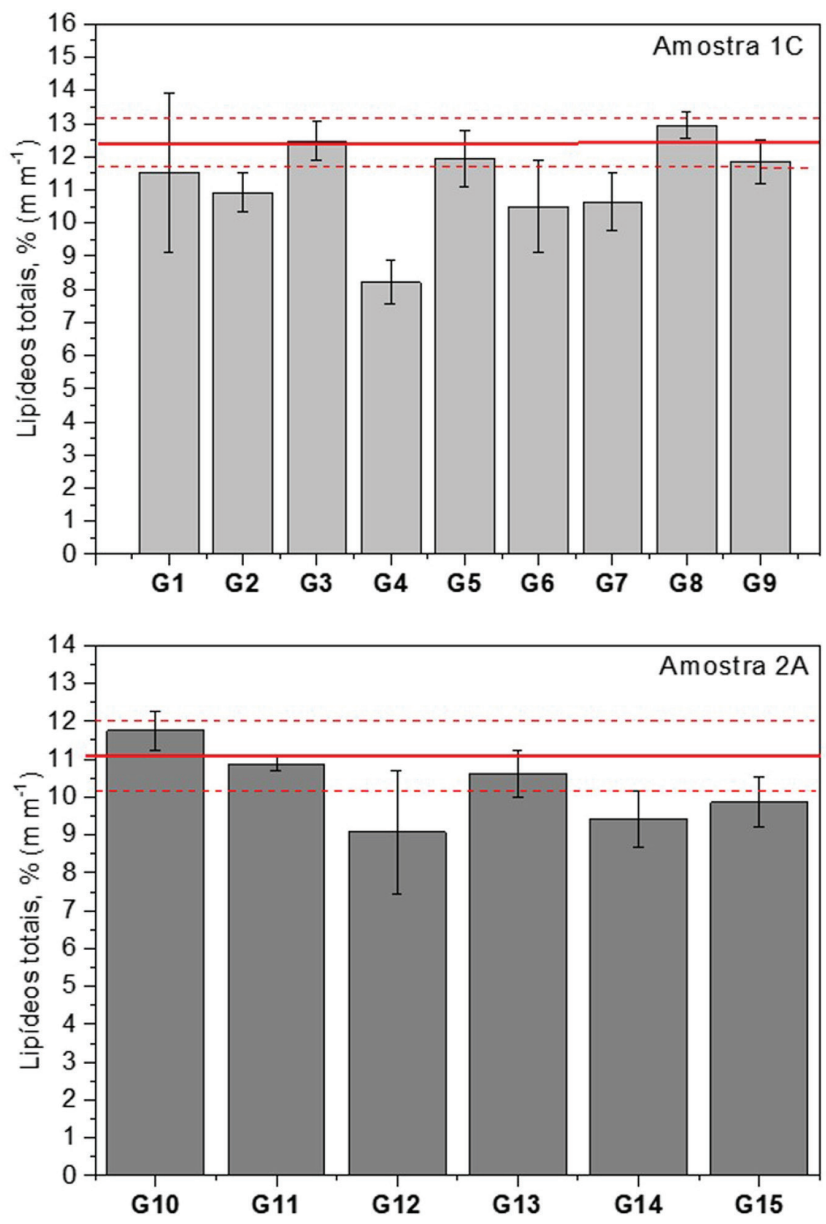

Figura 5. Valores de lipídeos totais $\left(\%, \mathrm{~m} \mathrm{~m}^{-1}\right)($ média \pm desvio padrão, $n=3$ ) obtidos pelos grupos (G1-G15) de estudantes para análise para amostra IC (valor de referência para lipídeos totais: $12,47 \pm 0,57 \%, \mathrm{~m} \mathrm{~m}^{-1}$, média \pm intervalo de confiança de $95 \%, n=3$ ) e amostra $1 \mathrm{~A}$ (valor de referência para lipídeos totais: $11,15 \pm 0,99 \%, \mathrm{~m} \mathrm{~m}^{-1}$, média \pm intervalo de confiança de $95 \%, n=3$ )

De modo geral, podemos observar que a maioria dos grupos obteve resultados com uma boa concordância com os valores de referência obtidos pelo método do IAL. Para a amostra $1 \mathrm{C}$, a qual foi analisada por 9 diferentes grupos, apenas 3 grupos não obtiveram resultados concordantes com os valores de referência. Assim, aproximadamente $66 \%$ dos grupos que realizaram o experimento usando UAE obtiveram teores de lipídeos totais, na amostra 1C, concordantes com o método oficial. A concentração de lipídeos da amostra 2A foi analisada por 6 diferentes grupos, sendo que todos os grupos chegaram a resultados com boa concordância com o valor de referência.

Considerando os 15 grupos que realizaram o experimento, apenas 3 não obtiveram resultados concordantes com os obtidos pelo método de referência, ou seja, $80 \%$ dos grupos que realizaram o experimento proposto para extração de lipídeos por UAE obtiveram valores satisfatórios. Os eventuais erros na realização deste experimento podem estar associados aos erros experimentais, alguns destes devido à falta de cuidado dos estudantes durante a transferência do solvente contendo os lipídeos para o béquer previamente pesado.

Neste experimento, os alunos foram avaliados por meio de questionários pré e pós-laboratório contendo questões associadas ao tema do experimento, além de um relatório contendo os resultados experimentais, cálculos e tratamento estatístico. De maneira geral, o feedback dos estudantes indicou que o experimento proposto foi bem recebido pelos alunos dos diferentes cursos de graduação, particularmente por possibilitar a determinação do teor de lipídeos em tempo reduzido e com menor consumo de reagentes. Este experimento, além de possibilitar aos estudantes a ampliação do conhecimento por meio da introdução de conceitos da UAE e da química verde, apresenta métodos alternativos simples e de baixo custo, que possibilitam a aplicação destes em aulas de laboratório, além de estimular discussões contemporâneas a respeito da ciência e o seu desenvolvimento em termos de produção de conhecimento.

\section{CONCLUSÃO}

O experimento desenvolvido com base na extração assistida por ultrassom (UAE) abordou conceitos elementares em análise química quantitativa e Química Verde, por meio de procedimentos alternativos aos convencionais para extração de lipídeos em amostras de alimentos. O procedimento proposto possibilitou a extração de lipídeos em amostras de bolachas com utilização de pequenos volumes de solvente extrator (15,0 mL de hexano) em um tempo reduzido de extração (30 min). Além disso, a comparação dos teores de lipídeos totais determinados pelo método usando UAE e pelo método oficial apresentaram uma boa concordância, comprovando a exatidão do método proposto.

O método UAE para extração de lipídeos em amostras de bolachas foi proposto como experimento de laboratório para um total de 15 grupos de alunos dos Cursos de Engenharia Química e Tecnologia de Alimentos. Dos alunos que realizaram o experimento, $80 \%$ obtiveram resultados satisfatórios e concordantes com os valores de referência para lipídeos totais nas amostras analisadas.

\section{AGRADECIMENTOS}

Os autores agradecem ao Conselho Nacional de Desenvolvimento Científico e Tecnológico (CNPq) (processos 444110/2014-3 e 421245/2018-2), à Coordenação de Aperfeiçoamento de Pessoal de Nível Superior (CAPES), à Universidade Federal de Santa Catarina e à Universidade Tecnológica Federal do Paraná pelo auxílio financeiro.

\section{MATERIAL SUPLEMENTAR}

Roteiro de experimento: Extração assistida por ultrassom (UAE) para determinação de lipídeos totais em amostras de bolachas, está disponível em http://quimicanova.sbq.org.br, na forma de arquivo PDF, com acesso livre. 


\section{REFERÊNCIAS}

1. Lisbôa, J. C. F.; Quím. Nova Esc. 2015, 37, 198.

2. Giordan, M.; Quím. Nova Esc. 1999, 10, 43.

3. Lenardão, E. J.; Freitag, R. A.; Dabdoub, M. J.; Batista, A. C. F.; Silveira, C. C.; Quim. Nova 2003, 26, 123.

4. Instituto A. L.; Métodos físico-químicos para análise de alimentos. Coordenadores Odair Zenebon, Neus Sadocco Pascuet e Paulo Tiglea. 4. Ed. São Paulo: Instituto Adolfo Lutz, 2008. 1020 p. Primeira edição digital.

5. Regitano-d'Arce, M.A.B.; Dissertação de Mestrado. Universidade de São Paulo, Brasil, 1991.

6. Brum, A. A. S.; Arruda, L. F.; Regitano-d'Arce, M. A. B.; Quim Nova 2009, 32, 849 .

7. Krug, F. J.; Rocha, F. R. P.; Métodos de Preparo de Amostras para Análise Elementar, 1 ${ }^{\mathrm{a}}$ ed., EditSBQ: São Paulo, 2016.

8. Chemat, F.; Rombaut, N.; Sicaire, A.; Meullemiestre, A.; Fabiano-Tixie; A., Abert-Vian, M.; Ultras. Sonochem. 2017, 34, 540.

9. Bendicho, C.; De La Calle, I.; Pena, F.; Costas, M.; Cabaleiro, N.; Lavilla I. T.; Anal. Chem. 2012, 31, 50.
10. Vilkhu, K.; Mawson, R.; Simons L.; Bates, D.; In. F. Sci. Emerg. Technol., 2008, 9, 161.

11. Kim, Y.-H.; Park, S.; Kim, M. H.; Choi, Y.-K.; Yang, Y.-H.; Kim, H. J.; Lee, S. H.; Biom. Bioenerg. 2013, 56, 99.

12. Li, H.; Pordesimo, L.; Weiss, J.; F. Res. Intern. 2004. 37, 731.

13. Khoei, M.; Chekin, F.; Food Chem. 2016, 194, 503.

14. Luque-García, J.; Luque de Castro, M.; J. Chrom. A 2004, 1034, 237.

15. Canteri, M. G.; Althaus, R. A.; Virgens Filho, J. S.; Giglioti, E. A.; Godoy, C. V.; Rev. Bras. Agrocomp. 2001, 1, 18.

16. Miller, J. N.; Miller, J. C.; Statistics and Chemometrics for Analytical Chemistry, $5^{\text {th }}$ Ed, Pearson Education Limited, 2005.

17. Martins, M. L.; Primel, E. G.; Caldas, S. S.; Prestes, O. D.; Adaime M. B.; Zanella R.; Sci. Chromatogr. 2012, 4, 35.

18. Rouhani, M.; Ind. Crops Prod. 2019, 132, 226.

19. Wen, C.; Zhang, J.; Zhang, H.; Dzah, C. S.; Zandile, M.; Duan, Y.; Luo, X.; Ultras. Sonochem. 2018, 48, 538.

20. Esclapez, M. D.; García-Pérez, J. V.; Mulet, A.; Cárcel, J. A.; Food Eng Rev. 2011, 3, 108 . 